\title{
Transcatheter aortic valve implantation: future directions
}

\author{
Volume 5 Issue 4 - 2016 \\ Isaac Pascual, Pablo Avanzas, Cesar Moris \\ Cardiac Catheterization Laboratories, Hospital Universitario \\ Central de Asturias, Spain
}

Correspondence: Cesar Moris, Cardiac Catheterization Laboratories, Area del Corazón, Hospital Universitario Central de Asturias, Oviedo, Spain, Email cesarmoris@gmail.com

Received: December 28, 2015 | Published: March 28, 2016 recent years the treatment of choice for degenerative aortic stenosis those at high surgical risk. ${ }^{1}$ This procedure has undergone a huge expansion in a short period of time, especially due to the excellent results published by different groups. The future evolution of this technique involves the so called "new indications" such as surgical treatment of degenerate biological valves, bicuspid aortic valves, aortic insufficiency and severe aortic stenosis with low or intermediate surgical risk.

\section{Degenerative biological valves}

Considering the large volume of patients over 65 years with aortic stenosis treated surgically with biological prostheses in the last years, treatment of degenerative biological valves is one of the main current and future areas of TAVI expansion. The degeneration may occur in both stenosis and regurgitation, although most often is mixed. The main mechanisms, which represent the leading causes of reoperation, are: calcification, fracture, pannus formation and valve thrombosis. Nowadays, reoperation represents the standard treatment for degenerate biological prostheses, involving a considerable risk in terms of morbidity and mortality (3\%). Reoperation mortality in patients at high surgical risk may exceed $20 \%$. In these patients, TAVI is an alternative and a much less invasive therapeutic option.

The initial preliminary data coming from registries as Canadian, Italian and German were very favourable for the reduction of the gradients and the decrease in the degree of aortic regurgitation. In the Global valve in valve registry, $93.1 \%$ of the procedures were successful, with a 30 -day mortality of $8.4 \%$ and the survival rate was $85.8 \% .^{2}$ Dvir D et al., ${ }^{3}$ raised and important issue, the size of the degenerated valve. In this study, prostheses were classified according to size: small $(\leq 21 \mathrm{~mm} ; 29.7 \%)$, intermediate $(>21$ and $<25 \mathrm{~mm}$; $39.3 \%)$ and large $(\geq 25 \mathrm{~mm} ; 31 \%)$. The factors significantly associated with mortality at one year were the small size of the prosthesis and the presence of stenosis, against regurgitation. ${ }^{3}$

The main risks of the valve in valve procedure are valve malposition and obstruction of the coronary arteries, more often in this context compared to native valves. Malposition of valve (movement of the device towards the aortic root or into the left ventricle) can reach $15 \%$ in this procedure. One related factor is the lowest radiopaque calcification of the bioprostheses compared to native valves. Furthermore, the positioning of the valve can be more difficult in those prostheses where its structure does not have a radiopaque stent. To lower this complication rate, the team's experience and a detailed analysis of each case are key aspects, as well as the knowledge of the architectural characteristics of each type of biological aortic prosthesis and the simultaneous use of transesophageal echocardiography during implantation. The incidence of coronary artery obstruction can reach $3.5 \%$. It represents higher frequency than that reported for TAVI in native valves, not exceeding $1 \%$. The mechanism of coronary occlusion is migration of a calcified valve cusp towards coronary ostium. It is usually more common in stenotic prostheses than in regurgitant ones. ${ }^{4}$ The carefully assessment of each case to identify those with a greater potential risk, is essential to minimize the risk of this serious complication, particularly those valves with the leaflets mounted on the outside of the frame.

TAVI is currently an alternative to surgical reoperation for degenerated aortic valve bioprostheses. Moreover, it can be the treatment of choice in high surgical risk patients. In the future we believe it will become the treatment of choice in this scenario for the vast majority of cases.

\section{Bicuspid aortic valve}

The treatment of aortic stenosis patients with bicuspid aortic valve (BAV) has been a subject of extensive discussion in recent years. Bicuspid aortic valve is the most common congenital heart valve disease, with $1-2 \%$ of estimated prevalence. The presence of BAV was an exclusion criterion in early studies of TAVI, but technological advances and greater accumulated experience, have opened new perspectives in this field. Currently, the existence of BAV is considered a relative contraindication for TAVI, especially in relation to the increased risk of residual aortic regurgitation. However, there have been series with good results with TAVI in selected patients with aortic stenosis and BAV and probably in the future TAVI will gain a greater role in this field. The BAV presents several morphological and structural aspects that should be taken in account when considering treatment with TAVI. The VAB ring usually has an extremely elliptical shape compared to the more oval morphology of the tricuspid aortic valve. It has also been described a very asymmetrical distribution of annular calcium in this type of valve.

These structural features have been associated with an increased risk of under expansion and malposition and higher proportion of paravalvular regurgitation and early valvular degeneration. In 
contrast, a number of studies have been published, reporting good valve performance in patients with BAV treated with TAVI. According to data from the German registry, the prevalence of BAV was $3 \%$, representing a total of 38 patients with BAV treated with TAVI, which is one of the largest series reported to date. There were no differences in either baseline characteristics or the success rate of the procedure. In the VAB group there was a higher proportion of significant aortic regurgitation, not related to 30 day mortality. The presence of BAV was not associated with higher mortality at one follow up.

In conclusion, the results of TAVI in BAV are encouraging; but we have to be cautious in their interpretation, as they come from small observational studies and therefore they should only be considered in this context. Technical advances in the design of new emerging devices including the ability to reposition the valve before final deployment, features to reduce paravalvular leakage, and the introduction of lowprofile delivery systems will allow individualizing and choosing the best valve in each case, and definitely will expand TAVI indications for aortic stenosis and BAV in the coming years.

\section{Intermediate surgical risk patients}

Although there is indirect evidence available from observational studies in patients with aortic stenosis and intermediate surgical risk, there is not information derived from randomized trials. The results of 2 randomized studies (SURTAVI and PARTNER II) will be available in the following years. They both compare the outcome of TAVI and aortic valve replacement in patients with severe symptomatic aortic stenosis and intermediate surgical risk (STS score between 4 and 8\%). The results of these studies will confirm whether TAVI is equivalent to aortic valve replacement in the treatment of these patients.

\section{Aortic regurgitation}

Several characteristics make valve implantation different in aortic regurgitation: non-calcified thin leaflets, large annulus size, dilated root, large regurgitant volume and dilation that may progress even after AVR. There is limited experience in inoperable patients with isolated aortic regurgitation treated with TAVI, although the preliminary results are acceptable. It is nevertheless essential to remark two variables: the high incidence of the need for a second valve and the degree of residual aortic regurgitation.

Roy et al., 5 included for TAVI with the Core Valve prosthesis a total of 43 inoperable patients with severe aortic regurgitation with mean EuroSCORE logistic and STS of $26.9 \pm 17.9 \%$ and $10.2 \pm 5.3 \%$ respectively. All patients had severe aortic insufficiency without aortic stenosis and 17 patients $(39.5 \%)$ had some degree of valvular calcification, documented by CT or echocardiography. The implant was successful in 42 patients $(97.7 \%)$ and 8 of them (18.6\%) required a second valve during the procedure by the presence of residual aortic regurgitation after the first implant, in all cases with absence of calcification. Final aortic regurgitation grade II or III was observed in 9 patients $(20.9 \%)$. At 30 days, the incidence of stroke and all-cause mortality was $4.7 \%$ and $9.3 \%$, respectively. After 12 months of follow up, all-cause mortality was $21.4 \%$.
It is necessary to accumulate more experience in this field, but surely the future development of new devices and the positive preliminary results, strongly suggest that the isolated aortic regurgitation can be also an area for future TAVI implementation.

\section{Other indications with future perspective}

There are certain clinical circumstances that greatly increase the preoperative risk but which are not covered by the standard rating scales. Entities such as liver cirrhosis, frailty or porcelain aorta, often represent a contraindication for surgical treatment of aortic stenosis. There is currently limited experience in dealing with these situations with TAVI, with good results in the short, medium and long-term follow-up up. ${ }^{6}$

\section{Conclusion}

TAVI has changed the treatment paradigm of aortic stenosis. It is the current treatment of choice for patients with severe symptomatic aortic stenosis considered inoperable and it is considered a valid alternative to surgery in those with high surgical risk. The excellent results, both due to the accumulation of experience and technical advances and better characterization and selection of candidates, suggest that in the near future the indication of TAVI should be extended to other scenarios. The results of the upcoming PARTNER II and SURTAVI studies will be critical to informing the field and expanding TAVI to intermediate-risk patients.

\section{Acknowledgments}

None.

\section{Conflicts of interest}

Authors declare that there is no conflict of interest.

\section{References}

1. Briales JH, García AJ, García JM. Percutaneous aortic valve implant: Current indications? Cardio core. 2010;45(2):53-57.

2. Dvir D, Webb J, Brecker S, et al. Transcatheter aortic valve replacement for degenerative bioprosthetic surgical valves: results from the global valve-in-valve. registry. Circulation. 2012;126(19):2335-2344.

3. Dvir D, Webb JG, Bleiziffer S, et al. Transcatheter aortic valve implantation in failed bioprosthetic surgical valves. JAMA. 2014;312(2):162-170.

4. Roy DA, Schaefer U, Guetta V, et al. Transcatheter aortic valve implantation for pure severe native aortic valve regurgitation. $J$ Am Coll Cardiol. 2013;61(15):1577-1584.

5. Bauer T, Linke A, Sievert H, et al. Comparison of the effectiveness of transcatheteraortic valve implantation in patients with stenotic bicuspid versus tricuspid aortic valves (from the German TAVI Registry). Am J Cardiol. 2014;113(3):518-521.

6. Pascual I, Avanzas P, Garcia AJ, et al. Implante percutáneo de la válvula autoexpandible Core Valve ${ }^{\circledR}$ en pacientes con estenosis aórtica grave y aorta de porcelana: seguimiento a medio plazo. Rev Esp Cardiol. 2013;66:775-781. 